\title{
Multiple Feedback Algorithm for RFID MAC Protocols
}

\author{
You-Chang Ko, Sumit Roy, Choong-Ho Cho, Hyong-Woo Lee, and Hari Krishna Garg
}

\begin{abstract}
This letter introduces a new tree-based anti-collision scheme using multiple feedbacks for uplink tag random access in a single-cell scenario. We examine MAC efficiency improvements that result from the proposed scheme in terms of uplink (UL) throughput for emerging radio frequency identification (RFID) networks, and compare it with the conventional tree based RFID MAC in ISO 18000-6 Type B standard.
\end{abstract}

Index Terms-RFID, anti-collision scheme, tree splitting algorithm, multiple feedback algorithm.

\section{INTRODUCTION}

$\mathbf{C}$ OLLISION resolution is a key component in RFID MAC protocols. Enhanced read throughput of tag IDs on the uplink is achieved via downlink (DL) feedback from the reader that contains information regarding successes/failures in (immediate) past read attempts, thereby allowing for quicker collision resolution. The two broad classes of MAC protocols to date - framed Aloha[4] and m-ary splitting algorithm (MSA) ${ }^{1}[1][2][3][5][6]$ - both generally use a single ternary type (Empty/Success/Collision) feedback symbol $(W)$ per packet access on a uplink (UL, tag-to-reader) slot. In this letter, we introduce multiple feedback algorithm (MFA) within a DMSA MAC $^{2}[3]$ protocol whereby the reader broadcasts multiple $(d \geq 2)$ feedback symbols for each UL packet access slot. Towards this end, a UL slot is extended beyond the packet payload to include the voting field. Fig. 1 illustrates the UL channel structure of the conventional schemes and our new MFA protocol. Ignoring preamble time the length of a UL time slot in conventional schemes $\left(T_{c \_s l t}\right)$ equals the packet duration $\left(T_{p k t}\right)$ as shown in Fig. 1-a). In contrast, the MFA UL slot $\left(T_{m_{-} s l t}\right)$ consists of two parts as in Fig. 1-b): $T_{p k t}$ and the voting field duration $(d \Delta \tau)$ containing $d$ mini-slots $\left(v_{1}, \ldots, v_{d}\right)$ with duration $\Delta \tau$ for each; an additional signal pulse placed at random within $d$ bins is sent in this period. The reader measures voting mini-slot corresponding to a data packet $S_{x}$. In case of a collision (i.e. multiple tags choosing the same UL slot), the slot is split subsequently into $m$ slots for collision arbitration as needed, where $m(\geq 1)$ is a variable chosen depending on the size of $d$. There are two distinct advantages of the MFA idea over ISO 18000-6 Type B (ISOB) standard[1] that uses a fixed splitting size (e.g. binary, $m=$ 2). Firstly, an estimate of the number of contending sources is available via observing the voting mini-slots corresponding

\footnotetext{
${ }^{1}$ It can be further classified into two categories depending on the sequence of collision resolution process: depth-first-search MSA(DMSA)[1][2][3] and breadth-first-search MSA(BMSA)[5][6].

${ }^{2}[1]$ and [2] are the special case of DMSA where $m=2$, a static binary splitting tree MAC, whereas [3] is the special case of DMSA where $m$ is dynamic.
}

to a UL data slot; this allows a choice of dynamic splitting size per slot and enhanced MAC efficiency. Secondly, even with multiple $(n \geq 2)$ access within a UL slot $S_{x}$ (i.e. a collision), some mini-slots will carry singleton signals with high probability implying that the corresponding data packet(s) is(are) guaranteed to be successfully received in the next retransmission. Cleary, larger $d$ implies that more accurate estimate of the number of contending sources is achieved. On the other hand, increasing the voting field will ultimately lead to throughput degradation as the overhead increased starts to negate any gains from the accurate estimate of collision events. The actual throughput is thus a function of the ratio $\gamma_{m}=\Delta \tau / T_{p k t}$, which was neglected in [3]. In this letter, we develop a new analytical model and examine the value of $d$ that optimizes UL throughput. The mathematical results are also supported by simulation results.

\section{Multiple Feedback Algorithm(MFA)}

A tag that transmitted its packet to a slot is supposed to send an additional signal pulse within one of $d$ mini-slot chosen at random. The reader broadcasts a feedback tuple $U, V$ at the end of each slot as follows: $U$ denoting the sensing result of $T_{p k t}$ duration, is the binary variable defined by

$$
U= \begin{cases}0, & \text { if Empty or Success } \\ 1, & \text { otherwise, }\end{cases}
$$

and $V$ is the sensing result of voting mini-slot, represented by a binary $d$-tuple $\left(V_{1}, V_{2}, \ldots, V_{d}\right)$ where

$$
V_{i}=\left\{\begin{array}{l}
0(\text { Empty }), \quad \text { if } v_{i} \text { is silent, or } \\
1(\text { Nonempty }), \quad \text { if } v_{i} \text { is not silent. }
\end{array}\right.
$$

Thus whenever $U=1$, the slot is split into $m=\sum_{i=1}^{d} V_{i}$ slots per the DMSA protocol, and the collided packets retransmitted in order within these $m$ slots per the $d$-bit sequence in $V$. Fig. 2 illustrates an example of collision resolution process in MFA with dynamic splitting. Suppose that four packets $-p k t_{1}, p k t_{2}$, $p k t_{3}$, and, $p k t_{4}$ - are to be sent in slot $S_{x}$ with three mini-slots $(d=3)$; two of them $\left(p k t_{2}\right.$ and $\left.p k t_{3}\right)$ send their voting signals to $v_{1}$ and the rest $\left(p k t_{1}\right.$ and $\left.p k t_{4}\right)$ to $v_{3}$ and $v_{2}$, respectively. The corresponding $\{U, V\}$ feedback for $S_{x}$ is then $\{1,(1,1$, 1) $\}$. In the next retransmission, $p k t_{2}$ and $p k t_{3}$ are supposed to compete again at $S_{x+1}$ : because the $\{U, V\}_{x+1}$ is $\{1,(1,0$, 1) $\}$ they can be identified in the following retransmission. Slot splitting is no longer required in $S_{x+2}, S_{x+3}, S_{x+4}$, and $S_{x+5}$ since $U=0$. In order to estimate the number of contending sources, consider the situation where unknown number $n(\geq$ 2 ) tags choose the same UL slot along with $d$ mini-slot voting 


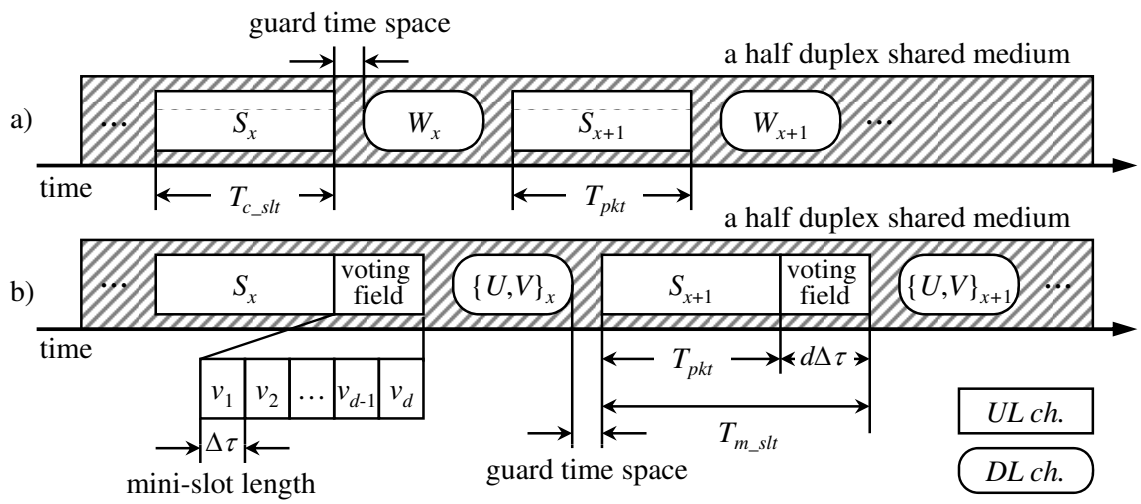

Fig. 1. UL time slot structure in conventional schemes a) and MFA b)

signals. The distribution of the $n$ voting signals in the $d$ minislots is therefore multinomial as (3).

$$
M\left(b_{1}, b_{2}, \ldots, b_{d} ; n\right)=\left(\begin{array}{c}
n \\
b_{1}, \ldots, b_{d}
\end{array}\right) \prod_{i=1}^{d} p_{i}^{b_{i}}=\frac{n ! d^{-n}}{\prod_{i=1}^{d} b_{i} !}
$$

where the $d$ bins contain $b_{1}, \ldots, b_{d}$ signals respectively such that $\sum_{i=1}^{d} b_{i}=n$ and $p_{i}=1 / d$ (each mini-slot is chosen equally). The first term in the middle equation is multinomial coefficient defined by $n ! / \prod_{i=1}^{d} b_{i}$ !. The number of all possible outcomes of the vector $\left(b_{1}, \ldots, b_{d}\right)$ is ${ }_{d} H_{n}\left(=_{d+n-1} C_{n}\right)$, i.e., the number with repetitions of arrangements of $n$ objects into $d$ bins. Let $N(n)$ denote the number of additional UL slots that are required to resolve an initial $n$-packet collision. Note that $N(0)=0, N(1)=0$ serve as initial conditions since there are no collisions with either 0 or 1 packet access in a slot. Let $X=\sum V_{i}$ from (2), the random variable taking values in the range $1,2, \ldots, d$. Then $N(2)$ can be deduced from (4), and thus $N(n)$ can be recursively computed as given by (5). For a given instance of $M(\cdot), X$ plus the additional number of slots required to resolve the subsequent collisions are consumed. Our delay derivation for MFA yields the same results as Appendix A in [3]. However because a UL slot time in MFA contains an overhead portion as illustrated in Fig. 1-b) we need to take the duration $(d \Delta \tau)$ into consideration to estimate the practical gains from MFA; hence $T_{m_{-} s l t}$ is represented by $T_{p k t}\left(1+d \gamma_{m}\right)$. Once selected, a tag transmits its packet at random within the initial UL frame length $\left(f_{0}\right)$ contributing to the initial packet loading factor $\left(\lambda_{0}\right)$, which

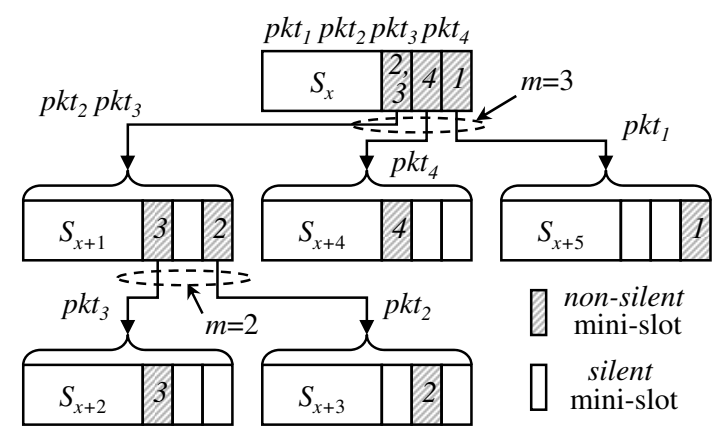

Fig. 2. An example of four packets collision resolution in MFA is defined by $n / f_{0}$, in each time slot; $\lambda_{0}$ can be represented by each value of the bins in (3) with $d=f_{0}$ at the start of an inventory session. Thus for a given system parameter set $\delta=\left\{n, f_{0}, d, \gamma_{m}\right\}$, the mean session latency in terms of the number of UL time slots is given by (6), and the normalized UL throughput ( $\left.\rho_{U L}\right)$ by (7). To get $\rho_{U L}$ for ISOB, $d$ and $\gamma_{m}$ are zero in $\delta$.

$$
\begin{aligned}
N(2)= & M(2,0, \ldots, 0 ; 2)[1+N(2)]+, \ldots,+ \\
& M(0,0, \ldots, 2 ; 2)[1+N(2)]+, \ldots,+ \\
& M(1,1, \ldots, 0 ; 2)[2+N(1)+N(1)]+, \ldots,+ \\
& M(0, \ldots, 1,1 ; 2)[2+N(1)+N(1)] \\
N(n)= & M(n, 0, \ldots, 0 ; n)[1+N(n)]+, \ldots,+ \\
& M(0,0, \ldots, n ; n)[1+N(n)]+, \ldots,+ \\
& M(n-1,1, \ldots, 0 ; n)[2+N(n-1)]+, \ldots,+ \\
& M(0, \ldots, 1, n-1 ; n)[2+N(n-1)] \\
= & \sum_{\substack{d b_{i} \leq n, 1 \leq i \leq d}}^{H_{n}}\left[M\left(b_{1}, \ldots, b_{d} ; n\right)\left\{X+\sum_{i=1}^{d} N\left(b_{i}\right)\right\}\right] \\
= & \left.\frac{E[X]+\sum_{b_{i} \neq n}^{H_{n}-d}\left[\frac{n ! d^{-n}}{\prod_{i=1}^{d} b_{i} !}\left\{\sum_{i=1}^{d} N\left(b_{i}\right)\right\}\right]}{1-d^{-n+1}}\right) \\
D(\delta)= & f_{0}+\sum_{0 \leq b_{j} \leq n,}^{f_{0} H_{n}}\left\{\frac{n ! f_{0}^{-n}}{\prod_{j=1}^{f_{0}} b_{j} !}\left(\sum_{j=1}^{f_{0}} N\left(b_{j}\right)\right)\right\} \\
\rho_{U L}= & \frac{\text { sum of successful packet time }}{\text { total UL times in a session }}=\frac{n}{D(\delta)\left(1+d \gamma_{m}\right)}
\end{aligned}
$$

\section{iII. Performance Evaluation}

We assume that packet and extra signal lengths are 16bit and 1-bit, respectively, i.e. $\gamma_{m}=6.25 \%$, and that reader channel sensing is sensitive enough to detect the presence of energy reliably within 1-bit mini-slot duration. We further assume that the channel or air interface is perfect, i.e. no packet losses - although this assumption may be unrealistic in real situation this letter simply investigates MAC layer gain from the proposed scheme. The lines and dots in Fig. 3 represent analytical results and simulation results, respectively. As seen in Fig. 3-a), a longer $d$ on one hand increases throughput but on the other hand contributes progressively to increased 
overhead; eventually there is no gain beyond $d=4$ within $\lambda_{0}$ $=4\left(f_{0}=4\right.$ and $\left.n=16\right)$. We note that prior to $\lambda_{0}=1(n=4)$ MFA is outperformed by ISOB since packets are much less likely to be collided and the presence of the voting field only increases overhead. However when collisions prevail beyond $\lambda_{0}=1$, gains are achieved depending on the size of $d$. The optimal $d$ for maximal $\rho_{U L}$ is found to be between $3-4$. The maximum gain from MFA is approximately $32 \%$ at $\lambda_{0}$ $=8\left(f_{0}=2\right.$ and $\left.n=16\right)$, which might be a probable situation because the reader doesn't know the size of $\operatorname{tags}(n)$ before a session starts, and has to blindly set $f_{0}$ via a rule of thumb, when compared with ISOB with $m=2$ as shown by Fig. 3b). As $f_{0}$ is increased the maximal UL throughputs in MFA and ISOB are asymptotically approaching to approx. 0.49 and 0.43 , respectively. We also note that throughput degradation in MFA as $\lambda_{0}$ increases is smaller compared to that in ISOB. Although not shown in the results, if $\gamma_{m}$ increases(decreases), the optimal $d$ decreases (increases), and so does the throughput gain. Fig. 3-c) illustrates how splitting size in MFA adapts dynamically to increased $\lambda_{0}$. The split size in MFA increases proportional to $\lambda_{0}$ adapting to slot based estimate, whereas it is fixed in ISOB regardless of $\lambda_{0}$.

\section{CONCLUSIONS}

We introduced a novel anti-collision principle - multiple feedback algorithm (MFA) - into RFID MAC and developed an analytical model to derive practical throughput as a function of the voting duration $d \Delta \tau$. MFA enables the reader to split the collided slot dynamically depending on the slot based backlogged tag estimate. We derived the optimal $d$ that yields the maximal UL throughput as a function of $\gamma_{m}$, ratio of minislot duration $\Delta \tau$ to packet duration $T_{p k t}$. With the optimal $d$, which is 4 at $\gamma_{m}=6.25 \%$, we showed that MFA achieves a significant throughput gain (32\%) at $\lambda_{0}=8$ relative to the conventional RFID spec. (ISOB)[1]. Since the real size of tag pool is unknown at the start, the initial tag loading factor $\lambda_{0}$ can be set conservatively; MFA is shown to be more robust than ISOB to this situation. In order to get DL gain, for a further study, MFA can be run on top of breadth-first-search $m$-ary splitting algorithm (BMSA)[5][6], whereby DL time is greatly reduced.

\section{REFERENCES}

[1] "ISO/IEC 18000-6: 2003(E), Part 6: Parameters for air interface Communications at 860-960 MHz", November 2003.

[2] J. I. Capetanakis, "Tree Algorithms for Packet Broadcast Channels", IEEE Trans. on Information Theory, Vol.25, No.5, September 1979.

[3] J-C Huang and T. Berger, "Delay Analysis of Interval-Searching Contention Resolution Algorithms", IEEE Trans. on Information Theory, Vol. IT-31, No.2, March 1985.

[4] C. Floerkemeier and M. Wille, "Comparison of Transmission Schemes for Framed Aloha based RFID Protocols", IEEE SAINTW 2006.

[5] Y-C Ko, S. Roy, J. R. Smith, H-W Lee, and C-H Cho, "RFID MAC Performance Evaluation Based on ISO/IEC 18000-6 Type C", IEEE Comm. Letters, Vol.12, No. 6, June 2008.

[6] Y-C Ko, S. Roy, J. R. Smith, H-W Lee, and C-H Cho, "An Enhanced RFID Multiple Access Protocol for Fast Inventory", IEEE Globecom, November 2007.

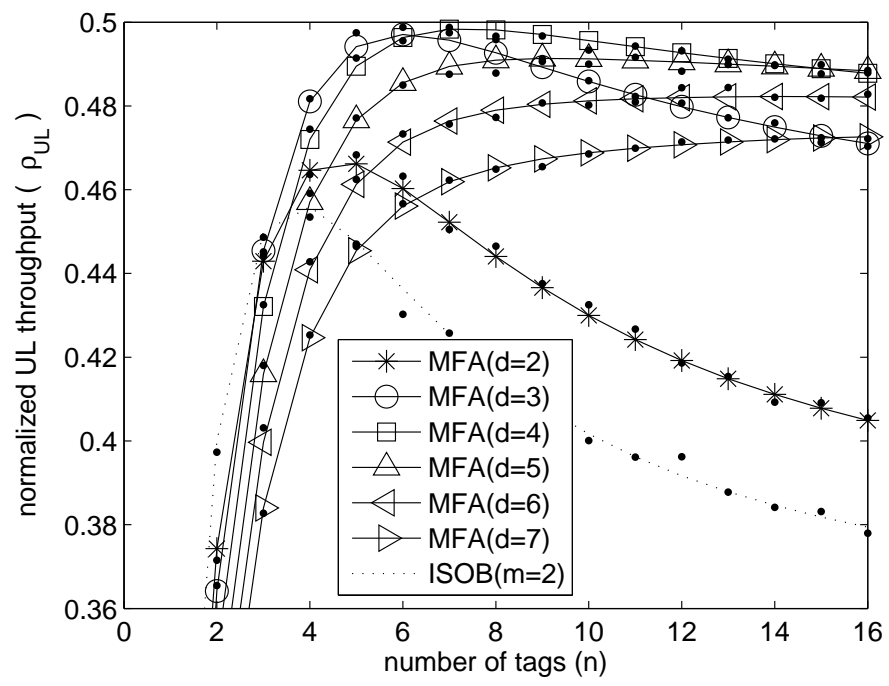

(a) UL throughput with $f_{0}$ (the initial UL frame size) $=4$ and $\gamma_{m}=6.25 \%$

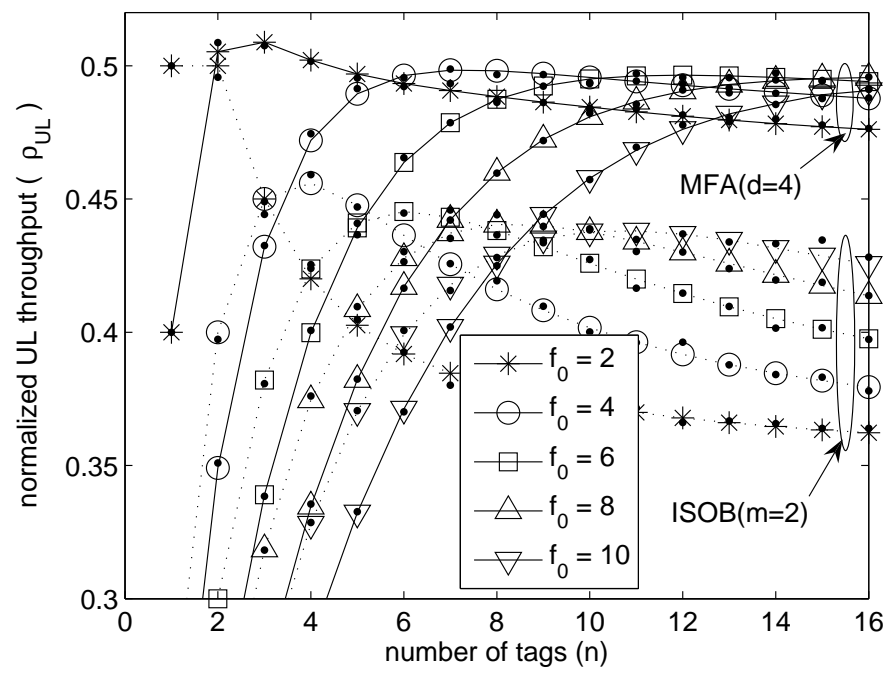

(b) UL throughput with $d$ (voting field size) $=4$ and $\gamma_{m}=6.25 \%$

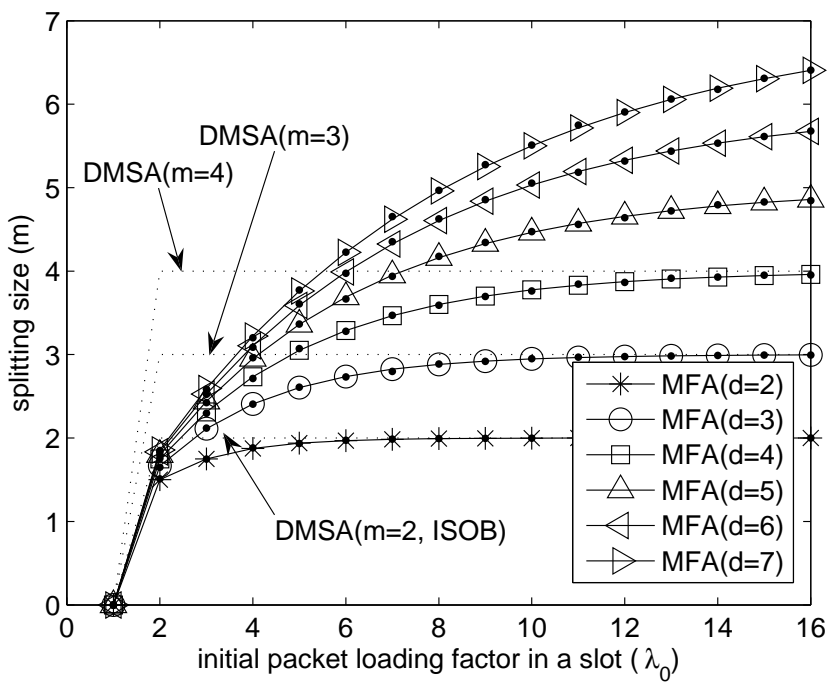

(c) Dynamic splitting size

Fig. 3. Performance evaluation comparison between MFA and ISOB 